\title{
Pulse oximetry monitoring: clinical implications
}

Lerma Hernandez, RN, CCRN, Staff Nurse, Department of Intensive Care Nursing, Andre V sale University Hospital, Montigny Le Tilleul, Belgium

Lerma Hernandez, 706 Rue de Goz e, 6110 Montigny Le Tilleul, Belgium. e-mail: lerma.hernandez@skynet.be

\section{INTRODUCTION}

Pulse oximetry monitoring was introduced well over a decade ago. It provides non-invasive measurement of arterial oxygen saturation $\left(\mathrm{SaO}_{2}\right)$ and is a valuable assessment tool, widely used to monitor respiratory status. It is becoming more accepted in clinical practice due to its ability to provide continuous monitoring of $\mathrm{SaO}_{2}$ and thus early warning of impending hypoxaemia.

However, if the nursing staff are to be able to respond appropriately and achieve the best possible patient outcome, they need to ensure that they are familiar with:

The technology of pulse oximetry

- Management of the patient with a pulse oximetry probe in situ

- How to rectify problems with the monitor and/or probe

- How to interpret the data provided.

\section{DESCRIPTION}

Pulse oximetry is a continuous, non-invasive method for monitoring the arterial oxygen saturation of functional haemoglobin $\left(\mathrm{SpO}_{2}\right)$. The patient's arterial oxygen saturation $\left(\mathrm{SaO}_{2}\right)$ level is determined by measuring the absorption (amplitude) of light waves as they pass through areas of the body that are highly perfused by arterial blood.

This is done using a sensor that has been placed over an area of the body containing a pulsating arteriolar bed, such as a finger, toe, bridge of the nose, or earlobe. The sensor contains two light-emitting diodes, one of which emits red light (wavelength, $660 \mathrm{~nm}$ ), while the other emits infrared light (wavelength, $940 \mathrm{~nm}$ ). The sensor also contains a photodetector, which measures the absorption of the light emitted by the diodes as the light passes through arterial blood (Figure 1).

The results obtained by pulse oximetry are based on the fact that oxyhaemoglobin, contains saturated haemoglobin and absorbs infrared light, while deoxyhaemoglobin contains desaturated haemoglobin and absorbs red light. The amount of infrared light absorbed will therefore depend on the amount of oxyhaemoglobin present in the blood and the amount of red light will depend on the amount of deoxyhaemoglobin. By comparing the amounts of infrared and red light absorbed, pulse oximetry is able to assess the proportion of oxyhaemoglobin to deoxyhaemoglobin in the patient's blood and thus measure the percentage saturation of arterial haemoglobin. For example if a patient has a pulse oximetry reading of $98 \%, 2 \%$ of the available haemoglobin is destaurated and $98 \%$ saturated.

\section{INDICATIONS}

There are two major indications for the use of pulse oximetry:

- As a warning system, pulse oximetry provides real-time indication of hypoxaemia and arterial desaturation.

- To judge the effectiveness of interventions. For example, an increase in arterial oxygen saturation indicates a positive effect for the addition of increased inspired oxygen.

Pulse oximetry is indicated whenever it is necessary to monitor continuously a patient's oxygenation status, thus providing an immediate, early warning of impending hypoxaemia. Examples of such clinical situations include:

-Weaning from mechanical ventilation

- During, and emerging from, anaesthesia

- Shocked states

- Chest trauma

- Pneumonia.

The accuracy of pulse oximetry readings is dependent on:

- The presence of a pulsatile flow

- Adequate functional haemoglobin

- The correct selection and placement of the sensor.

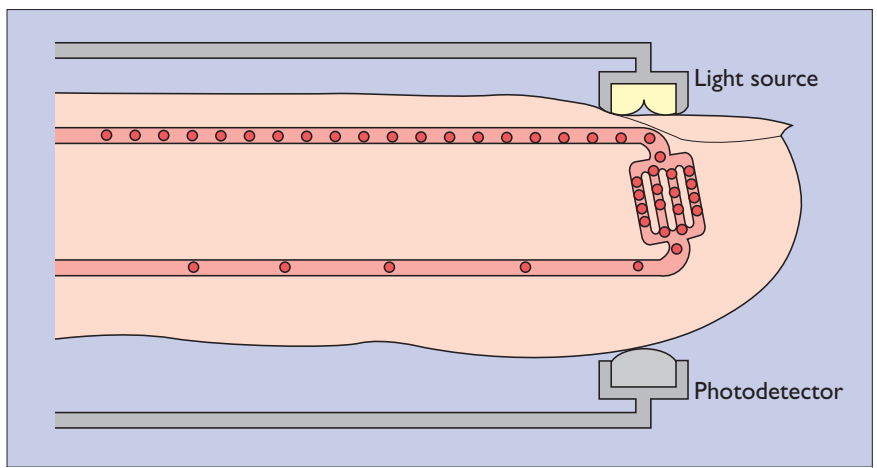

Figure 1. Components of sensor. (Reproduced with permission from Nellcor ${ }^{\circledR}$, part of Tyco Healthcare.) 


\section{SENSOR SITES}

The sensor sites are illustrated in Figures 2-5.

\section{FACTORS AFFECTING PULSE OXIMETRY READINGS}

The use of injectable dyes, e.g. methylene blue, affects the transmission of light and therefore the $\mathrm{SpO}_{2}$ readings. Although bilirubin, even at high levels, does not affect pulse oximetry, skin pigmentation can have an effect leading to greater inaccuracy in dark-pigmented individuals.

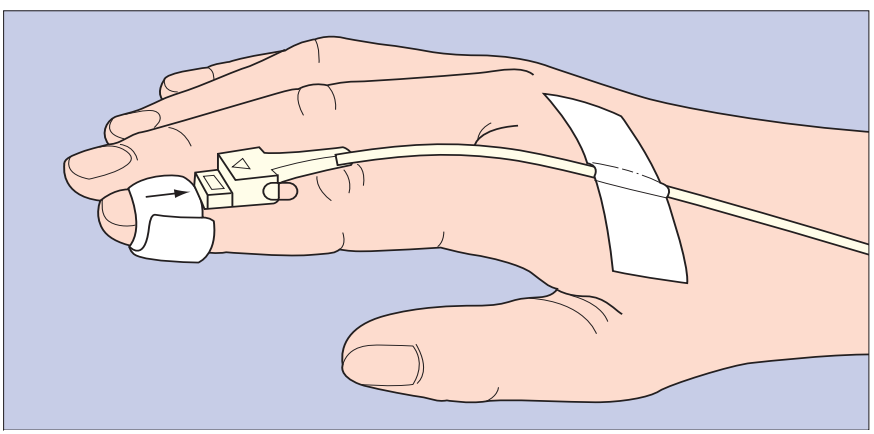

Figure 2. Sensor in position on finger. (Reproduced with permission from Nellcor ${ }^{\circledR}$, part of Tyco Healthcare.)

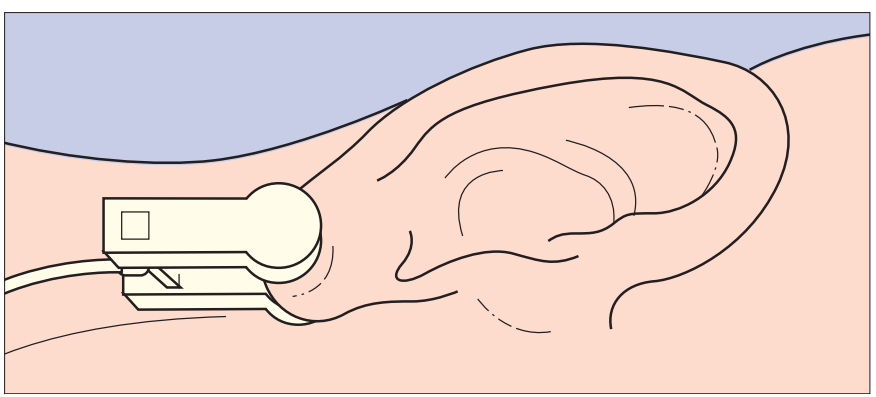

Figure 3. Sensor in position on earlobe. (Reproduced with permission from Nellcor ${ }^{\circledR}$, part of Tyco Healthcare.)

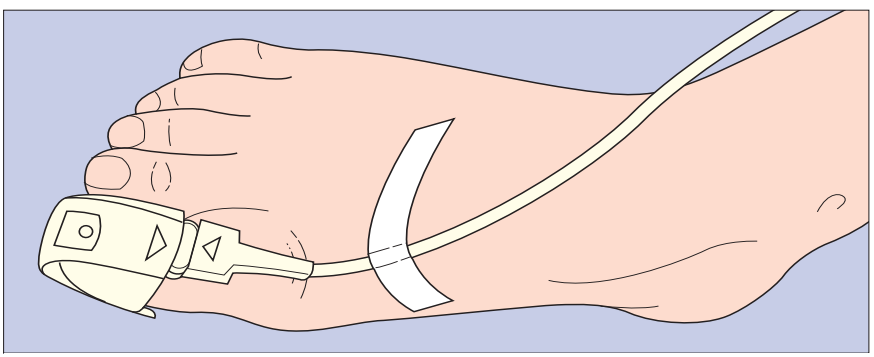

Figure 4. Sensor in position on toe. (Reproduced with permission from Nellcor ${ }^{\circledR}$, part of Tyco Healthcare.)

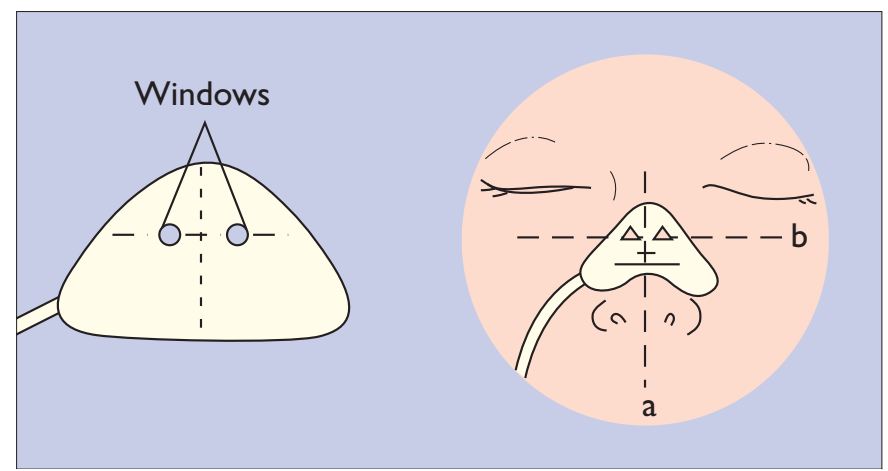

Figure 5. Sensor in position on nose. (Reproduced with permission from Nellcor ${ }^{\circledR}$, part of Tyco Healthcare.)
Optical interference, such as excessive ambient light reaching the photodetector, can affect readings by interfering with the transmission of light from the emitting sources to the photodetector.

Some nail polishes block the transmission of light through the pulsatile bed; colours that are particularly problematic include black, dark blue and green. Optical shunting can occur in patients who have synthetic finger nails in situ, with the light passing through the nail rather than through the pulsatile bed.

\section{TIPS FOR ENSURING ACCURATE READINGS}

A pulsatile flow is difficult to detect in patients who are poorly perfused as a result of hypotension, cardiac arrhythmia or hypothermia. In these cases, a central sensor site should be selected, such as the bridge of the nose, as it is more likely that a flow will be detected.

Pulse oximetry is inaccurate in the presence of abnormal haemoglobin. For example, carboxyhaemoglobin will result in false high readings and methaemoglobin in false low readings.

Patient movement often results in artefact and false alarms due to the difficulties of the pulse oximeter in identifying a flow. In patients who are confused or agitated, a central site such as the bridge of the nose may reduce the amount of movement experienced by the sensor and therefore increase the accuracy of the reading. Some pulse oximeters have the ability to block out movement artefact, while in others it is possible to increase the 'averaging time', which is the time the monitor has to determine a reading.

The sensor should be protected form excessive ambient light; if a finger sensor is used, placing a towel over the hand will black out any external light.

Venous congestion or pulsation can occur when sensors are applied too tightly or additional tape used to fix the sensor. This can also occur in patients who have congestive cardiac failure. Due to the constriction or congestion, a pulse is created in the venous circulation.

In addition, the pulse oximeter cannot distinguish between an arterial and venous pulse. A sensor should be applied snugly but not too tightly.

\section{CONCLUSION}

Clinically, the use of pulse oximetry for non-invasive monitoring of arterial oxygen saturation is an important monitoring tool in the care of critically ill patients. Although many factors can affect the accuracy of pulse oximetry, the technique has become a standard of care and is widely applied in intensive and highdependency care. It has important potential for improving the quality of patient outcome and helping both nursing and medical care givers.

\section{FURTHER READING}

Boggs RL et al. (1995). Monitoring of arterial oxygen saturation. In: ACCN Procedure Manual. $3 r d$ ed. Philadephia: WB Saunders: 359.

Darovic G. (1995). Continuous monitoring of arterial oxygen saturation. In Hemodynamic Monitoring: Invasive and Non-Invasive Clinical Application. 2nd ed. Philadelphia: WB Saunders: 355.

Dantzker et al. (1995). Measurement of arterial oxygenation. In: Comprehensive Respiratory Care. 1st ed. Philadelphia: WB Saunders: 255-256.

Grossbach I. (1993). Case studies in pulse oximetry monitoring. Critical Care Nurse; 13: 63-65.

Loeb S. (1994). Respiratory care. In: Manual of Critical Care Procedure. Springhouse: 249-252.

Thelan RD et al. (1994). Pulmonary therapeutic management. In: Critical Care Nursing. 2nd ed. Mosby: 454-455. 